\title{
Exploring and Comparing the Performance of Design Methods Used for Information Intensive Websites
}

\author{
Tarannum Zaki1 ${ }^{*}$, Zinia Sultana², S M Anisur Rahman ${ }^{3}$, and Muhammad Nazrul Islam ${ }^{4}$ \\ Department of Computer Science and Engineering, Military Institute of Science and Technology (MIST), Dhaka, Bangladesh \\ emails: *1tarannumzaki@yahoo.com; ${ }^{2}$ sultana.hiramony@gmail.com; ${ }^{3 a n i s 972 @ g m a i l . c o m ; ~ a n d ~}{ }^{4}$ nazrul@cse.mist.ac.bd
}

\section{ART ICLE INFO}

\section{Article History:}

Received: 29th February 2020

Revised: $10^{\text {th }}$ May 2020

Accepted: $12^{\text {th }}$ May 2020

Published online: 21 st July 2020

\section{Keywords}

Human-computer interaction (HCI) Interactive dialogue model (IDM)

User experience (UX)

Card sorting

Usability

\begin{abstract}
A B S T R A C T
At present, web applications are one of the most widely used software applications in different organizations. A website is a virtual representation of the structure of any organization. Good user experience of a website is highly dependent on the content organization or design of a website. Thus, the design is a very important factor to develop a high usability standard website. Different types of design techniques are used to develop different types of websites. It is a very challenging task to develop information-intensive websites using a suitable design technique so that the website can serve its intended purpose with enhanced usability and user experience. Therefore, the objective of this research is to compare the performance of two alternate design techniques for developing an information-intensive website. To achieve this objective, the existing design techniques were explored; and selected the Card Sorting and Interactive Dialogue Model (IDM) techniques to design two websites for an orphanage system. Later, the developed orphanage web systems were evaluated with ten participants through a user study; and found that the IDM design technique performs better than the Card Sorting technique in terms of the system's usability and user experience.
\end{abstract}

(C) 2020 MIJST, All rights reserved.

\section{INTRODUCTION}

Design refers to a draft model construct or planned before developing the final product that concerns how the contents will be organized and different functions will be performed (Preece et al., 2004). System behavior is generally reflected upon the design of that system since the design is not the final product, but it determines how the final product will function or work.

Again, the design has an impact on a system's usability and user experience. Since the quality of different softwareoriented applications such as web applications, mobile applications or any other software applications are highly dependent on their usability and user experience factors. Practitioners thus concern about the design technique considered to develop a mobile or web system to improve its usability and user experience (UX) (Islam et al., 2020). The usability of a website primarily depends on some characteristics, for example, understanding the needs and expectations of users, easy and user-friendly navigation, clear requests to specific actions, complete content organization, consistent interface and operation, that is, the effectiveness, efficiency and satisfaction (Abran et al., 2003; Rabi u et al., 2012; Zhang et al., 2010). A clear concise and intuitive user interface (UI) design may lead to achieving these characteristics for a website. Thus, the design is a crucial activity to develop software systems with enhanced usability and UX.

There are several design techniques to design and develop websites such as HDM (hypertext design model), IDM (interactive dialogue model), Card Sorting, UML (unified modeling language), WSDM (website design method), SIDE (Semiotic Interface sign Design and Evaluation) etc. (Bolchini \& Paolini, 2006; Garzotto et al., 1993; Mubin et al., 2015; Troyer \& Leune; 1998; Spencer, 2009; Islam \& Bouwman, 2016). Thus, it is a very crucial concern for HCI (human-computer interaction) practitioners to understand which design approach is better and easy to follow. Though, many comparative studies are existing on HCI that focused on comparing the performance of different usability and UX evaluation techniques. But no study has been carried out focusing on the comparison among the design techniques used to develop web applications. Therefore, the objective of this research is to investigate and compare the performance of two alternate design techniques to design and develop web applications. To attain this objective, an example web application for the orphanage management system is designed and developed using the IDM and the Card Sorting techniques followed by an evaluation study that was replicated with 10 participants in a laboratory environment. 
To present the rest of the paper, it is organized as follows. Section 2 provides the theoretical concept of IDM and Card Sorting design techniques as well as some related works regarding the focused issue. The study methodology is depicted in Section 3. Section 4 illustrates the system design and development. Section 5 discusses the evaluation study including participants' profiles, study procedure, and data analysis and findings. Finally, Section 6 discusses the study outcomes highlighting the research contributions followed by limitation, future work and a brief concluding remark.

\section{BACKGROUND THEORY AND LITERATURE}

This section is divided into three parts where the initial two parts briefly discuss the theoretical concept of the two design techniques - IDM and Card Sorting and the final part describes some related research works and development published during the past few years.

\section{A. Interactive Dialogue Model (IDM)}

IDM or interactive dialogue model is a design technique focused on possible conversation exchange between users and the content of an application to identify and organize the user requirements (Bolchini \& Paolini, 2006). It is formally known as a dialogue-based model, because the features of an application are not designed based on some particular predefined design architecture rather the design is crafted based on the user interaction with that specific application which is referred to as dialogue. IDM is considered to be a convenient approach for multi-channel applications (Bolchini \& Paolini, 2006) (e.g. embedded system, web applications, android apps and so on) which means it has a fruitful result in providing similar user experience for identical content regardless of cross-platform environment. It is widely used for applications which are highly informative and interlinked and not taken into account for applications that contain a large number of operations. IDM is performed using three consecutive steps - C-IDM (conceptual IDM), LIDM (logical IDM) and P-IDM (page IDM) (Bolchini \& Paolini, 2006). C-IDM is channel independent and used to identify the main topics or concepts of a website and a necessary relation between the topics. L-IDM is channeldependent and used to identify a number of dialogue units within a specific topic for a particular development platform. $\mathrm{P}$-IDM is the page design or prototype of each topic and required navigational links. In general, the interactive dialogue model is a useful, natural, inexpensive, userfocused but a bit effortful design technique and applicable for all types of applications developed in different platforms (Bolchini \& Paolini, 2006).

\section{B. Card Sorting}

Card Sorting is a design technique used to build the information architecture of an application, that is, how the content of an application is identified, categorized and organized in accordance with users' convenience by labeling and classifying the entire content of an application (Spencer, 2009). This design technique is mostly used for web applications that are rich in information, traversable but less operative and not considered as a convenient approach for multi-channel applications. A group of participants usually perform a Card Sorting session (Spencer, 2009) where they use a number of small colorful cards. All the participants individually identify the user requirements of the application (i.e. what should be on the homepage, the hierarchy of content and the navigational structure etc.) by labeling the cards accordingly and further grouping them into different categories. The hierarchy of different topics might be interpreted using cards with different colors. This design technique eventually helps to design an application where similar user requirements can be recognized with a lot of variation. This Card Sorting session can be also performed using different software tools available online. It is indeed a very simple, inexpensive, widely used and user-centered design process that used mainly to design the structure of web applications regarding how the content will be organized but may not provide a strong analysis regarding the end to end navigation process.

\section{Related Works}

Previously, different hypertext applications used to be designed using the "page by page" design approach (Bolchini \& Garzotto, 2008), that means an application containing several interconnected links were developed by designing a particular page and then moving on to the next hyper-linked page. This approach is usually unable to reflect the user requirements completely as designing an individual page successively results in a partial content representation of an application. As a result, to fulfill the user requirements for interactive hypertext applications, HDM (hypertext design model) has been introduced (Garzotto et al., 1993), where the authors have presented a design and browsing schema that help to understand how the user requirements can be organized in a user interactive way by classifying the requirements into different entities and structural links from different perspectives. Next, to deal with the increasing and complex user requirements, the IDM has been proposed in (Bolchini \& Paolini, 2006; Bolchini \& Garzotto, 2008). Bolchini and Paolini (2006) highlighted the usage of IDM for designing multi-channel applications. Perrone et al. (2005) have introduced a "Stakeholders Centered Approach" and showed that IDM is an effective design model that supports user requirements and goals. Some academic projects have been carried out on interactive and contentintensive applications at Indiana University by Craske (2010). Spagnolo et al. (2010) integrated the concept of IDM with the concept of rich internet applications (RIA) to propose a new design strategy named SEE-IA (SEarch Enhanced Information Architecture). Coppola et al. (2015) proposed the Mobile-IDM as the extension of the IDM technique and the usages of Mobile-IDM are discussed through a case study of designing a tourism mobile application. In another study, Islam et al. (2017) showed the applicability of the IDM design technique to design and develop a multi-channel military application to facilitate communication between the higher commanders and the front-line troops during a military operation. Thus, along with multi-channel features, IDM design technique has received much acceptance for interactive and contentintensive applications.

Again, among all the traditional techniques available for designing interactive applications, Card Sorting is one of the widely used ones. Rugg and McGeorge (2005) have discussed different sorting techniques. For example, card sorts to identify user requirements for any application. The authors have also given necessary guidelines for choosing sorting techniques along with an example of a sorting session and concluded that sorting techniques are a useful part of requirements analysis. Card Sorting has gained popularity for designing information-intensive applications. 
Zimmerman and Akerelrea (2002) have summarized the Card Sorting technique and verified the derived classification for informative websites. They have also suggested an approach towards it and concluded that Card Sorting techniques must be enhanced using social science concepts. Card Sorting can be conducted physically or using online software, while the effectiveness of its result using either approach has been compared (Petrie et al., 2011). The comparison has been carried out on two types of websites museum and news and found that the information architecture of the respective website drawn using card sorting differs from the typical ones depending on the cultural differences of the participants.

Another study has been conducted by Faiks and Hyland (2000) using the Card Sorting technique to identify and organize the concepts of an online library system, and then showed that this design technique is useful enough before developing an application as the concepts are gathered from a users' point of view. The card sorting technique is used in a number of other studies to design the websites, including a website for the electronic banking system (French et al., 2007), Utah State University's library website (Duncan \& Holliday, 2007), e-commerce websites in Kuwait (Al-Fadhli, 1997), websites for the health care worker and the general public (Wentzel et al., 2016), and usable website for an aging population (Kurniawan \& Zaphiris, 2007). Again, card sorting is also used to evaluate the usability and information architecture of web systems (Tullis, 2007; Ebenezer, 2003).

A suitable and effective design technique for a particular application depends on evaluating some usability factors such as its content organization, user-friendly navigational structure, performance, responsiveness and on the likes (Kumar \& Goundar, 2019; Razzak \& Islam, 2020). These usability factors or parameters are measured through a number of existing Usability Evaluation Methods (UEM) that includes, for example, task-analysis, heuristic evaluation, think-aloud, interviews, cognitive walkthrough, etc. (Park et al., 2020; Sagar \& Saha, 2017; Palmer, 2002; Bolchini \& Garzotto, 2007).

These methods have been compared with each other to assess the performance of various usability evaluation methods in (Matera et al., 2002; Ternauciuc \& Vasiu, 2015; Jeffries et al., 1991; Karat et al., 1992; Gray \& Salzman, 1998; Brejcha \& Marcus, 2013; Marcus, 2015). For example, two analytical evaluation methods were compared in (Matera et al., 2002), where an educational computer game developed for young children was evaluated by two different evaluation methods and then their performances were compared. Similarly, Conte et al. (2007) proposed a usability evaluation technique for web application and then compared its performance with Nielsen's heuristics.

Based on the above discussion, it was found that the earlier work mainly discussed the procedure of applying the design techniques with example cases, assessing the performance of a design technique to design and develop different types of software systems, and how a design technique can improve the system usability to end-users. On the other hand, in HCI, several studies focus to compare the performance of different usability evaluation methods (UEMs) in a different context. But none of the studies is yet conducted to compare the performance of different design techniques in respect of system usability and UX. Usability and UX are strictly related to system design. Therefore, it is of utmost importance and an open issue to explore whether choosing a specific design technique provides any impact on systems' usability and UX. Similarly, it is not yet revealed which kind of design technique would be a better choice for UI practitioners to achieve enhanced usability and UX for information-intensive websites.

\section{METHODOLOGY}

The research was carried out by following three phases as shown in Figure 1. Firstly, a number of design techniques were identified through a literature survey. Each design technique has its own merits and demerits. The features of design techniques have been explored and found that only a few are explicitly focused to design information-intensive multi-channel (web and mobile) applications. The objective of this research is to observe the performance of different design techniques and thus, this research has preliminarily selected the IDM and Card Sorting design techniques. Among a few others, both the Card Sorting and IDM are old and widely used to design information intuitive web application. Again, designing information-intensive websites mainly focuses on the content and navigation structure and the selected design techniques are addressing the same issues. Moreover, both the card sorting and IDM techniques are focusing on the end-users and their requirements; and not dependent on some predefined design model.

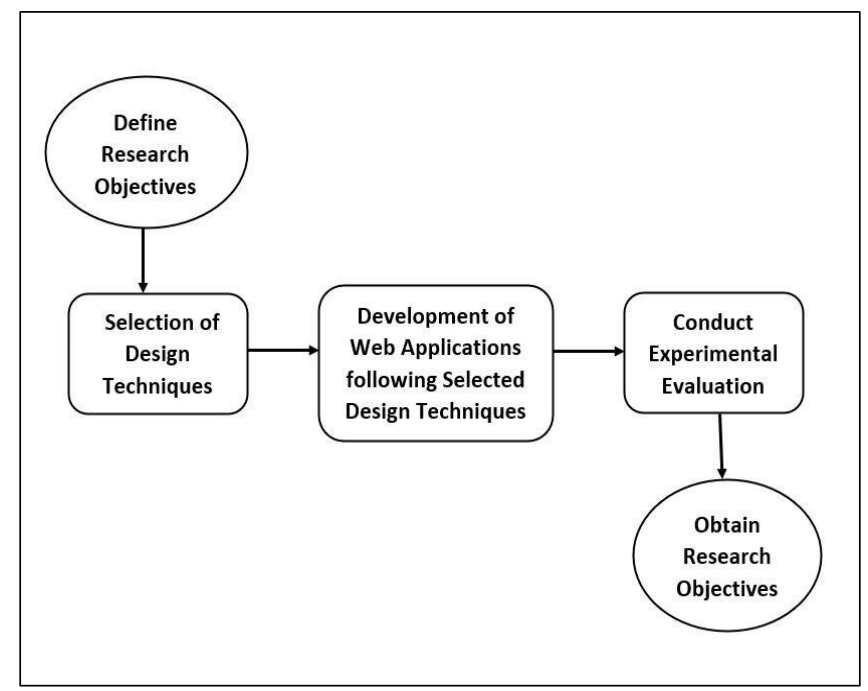

Figure 1: Phases of research methodology

Secondly, two websites for an orphanage management system were designed and developed following the selected design techniques. The orphanage system can be considered as an information-intensive website and presumed to provide information regarding different orphanages and accommodated orphans worldwide, adoption and admission information and processes, donation information, and other relevant online services.

Finally, a within-subject experiment was conducted to compare the performance of design techniques in terms of the usability and user experience of the developed systems. A number of participants were recruited and asked them to perform a set of tasks for both websites. During the experiment a number of metrics have been measured and analyzed using descriptive statistics and qualitative approach. A more detailed discussion of the study procedure and data analysis is presented in Section 5. 


\section{DESIGN AND DEVELOPMENT}

Two web portals for the orphanage system were designed using the Card Sorting and IDM techniques. A total of four designers participated to design and develop the web portals. All participants (designers) were graduated in Computer Science, highly expert in the respective design techniques, had practical experiences of applying the design techniques in real-world projects, all had teaching and research experiences in the field of human-computer interaction. Each portal was designed by two designers following either IDM or Card Sorting design technique. However, before starting the design task, all participants discussed together to understand the objectives and requirements of an orphanage system so that participants can design the orphanage system based on a common paradigm related to system requirements, and to reduce the effect of confounded variable.

\section{A. Design Using Card Sorting}

An open Card Sorting technique (Garzotto et al., 1993) is used to design the orphanage system, where participants of Card Sorting make assumptions on the content of a particular website theme and label them into a particular group. At first, they individually labeled different topics of the content on a number of small cards, then grouped altogether into different clusters and finally organized the subgroups into required clusters. A total of nine primary groups were found, that includes: About Us, Orphanage Info, Orphan Info, Contribute Now, Admission, Adoption, Site map, Privacy Policy and FAQ. Each group consists of several construct or topic, for example, s About Us consisted of Mission \& Vision, Our Service, Board of Directors and Contact. Orphanage Info consisted of an Orphanage List, that contained a number of orphanages, while each orphanage is represented through its' Name, Contact, Photos, Brief Introduction and Latest Donation News. Similarly, the
Contribute Now consisted of Donation Form and Volunteer Form, while the Adoption consisted of Eligibility Document and Adoption Form. The overall information architecture derived from the Card Sorting technique is presented in Figure 2.

\section{B. Design Using IDM}

The IDM-based design is discussed in three steps, namely CIDM, L-IDM, and P-IDM.

\section{i. Conceptual IDM (C-IDM)}

The first phase is C-IDM and does not depend on the channel or platform type. Conceptual IDM consists of three design parameters (Bolchini \& Paolini, 2006) - topic, relevant relation, group of topics and a parametric group of topics (optional). The topic is of two types - single topic and kind of topic. A single topic is a concept which can be an issue of an interactive discussion between human and web application and does not contain multiple instances in the application. In the orphanage portal website, single topics were About Us, Contact Us, Admission, Donate Now, Online Counseling, Site map, FAQ and Privacy Policy. For example, About $U s$ was a single instance and only contained some introductory information. Kind of topic is the same as a single topic except that it contains multiple instances in the application. In this website, the topics covered included Explore Orphanage, Donation Report, Know Orphans and Adoption Form. For example, Explore Orphanage had multiple instances because it contained some information of a number of orphanages. Next, relevant relation only exists for a kind of topic and indicates the change of conversation from one kind of topic to another with necessary cardinality (1:1, 1: $n$, etc.). Again, there are five relevant relations showed in this design (see Figure 3), for example, Explore Orphanage is related with Donation Report through "provides", while, Explore Orphanage is related with Know Orphans through

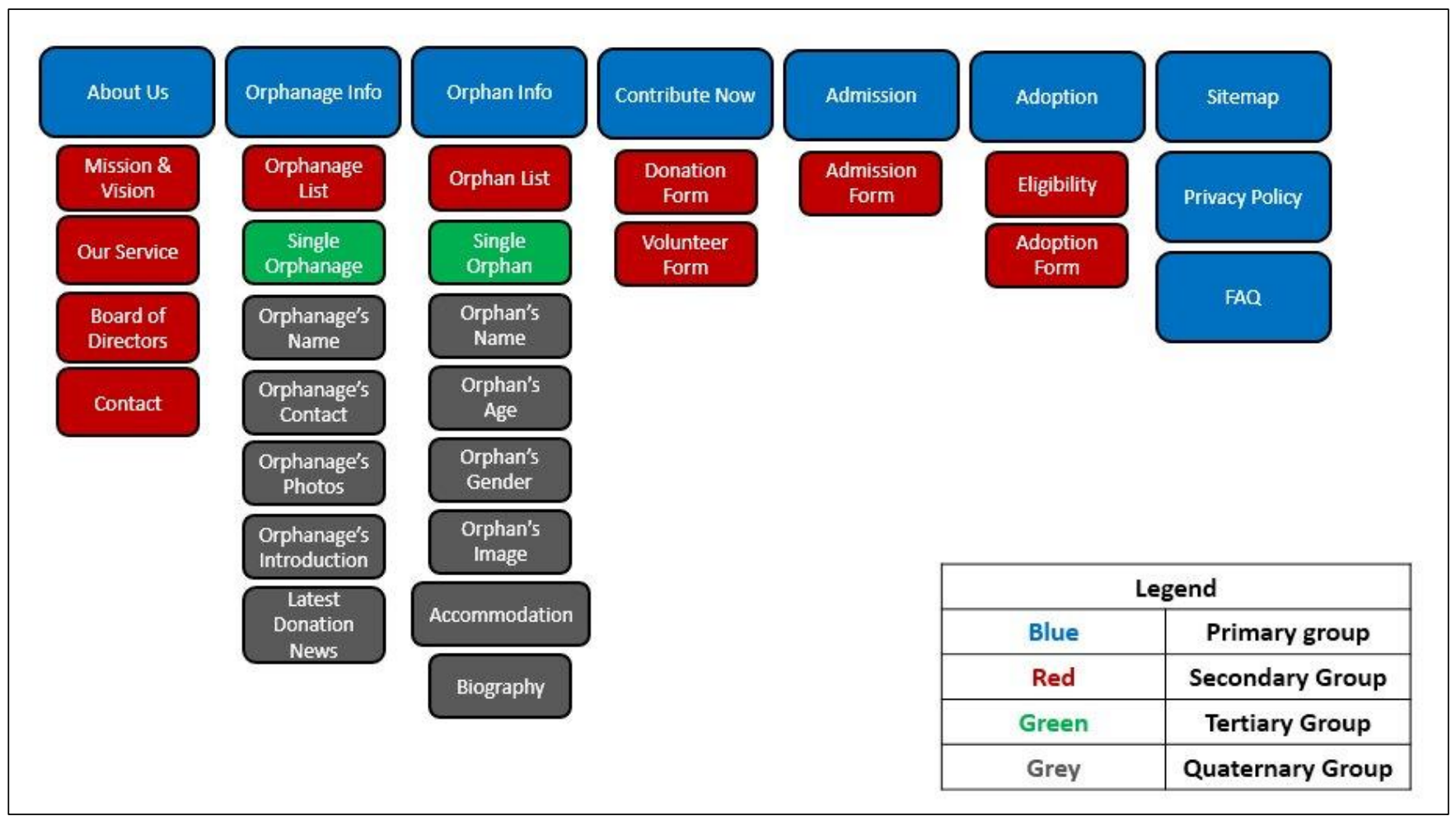

Figure 2: Card sorting for orphanage portal 


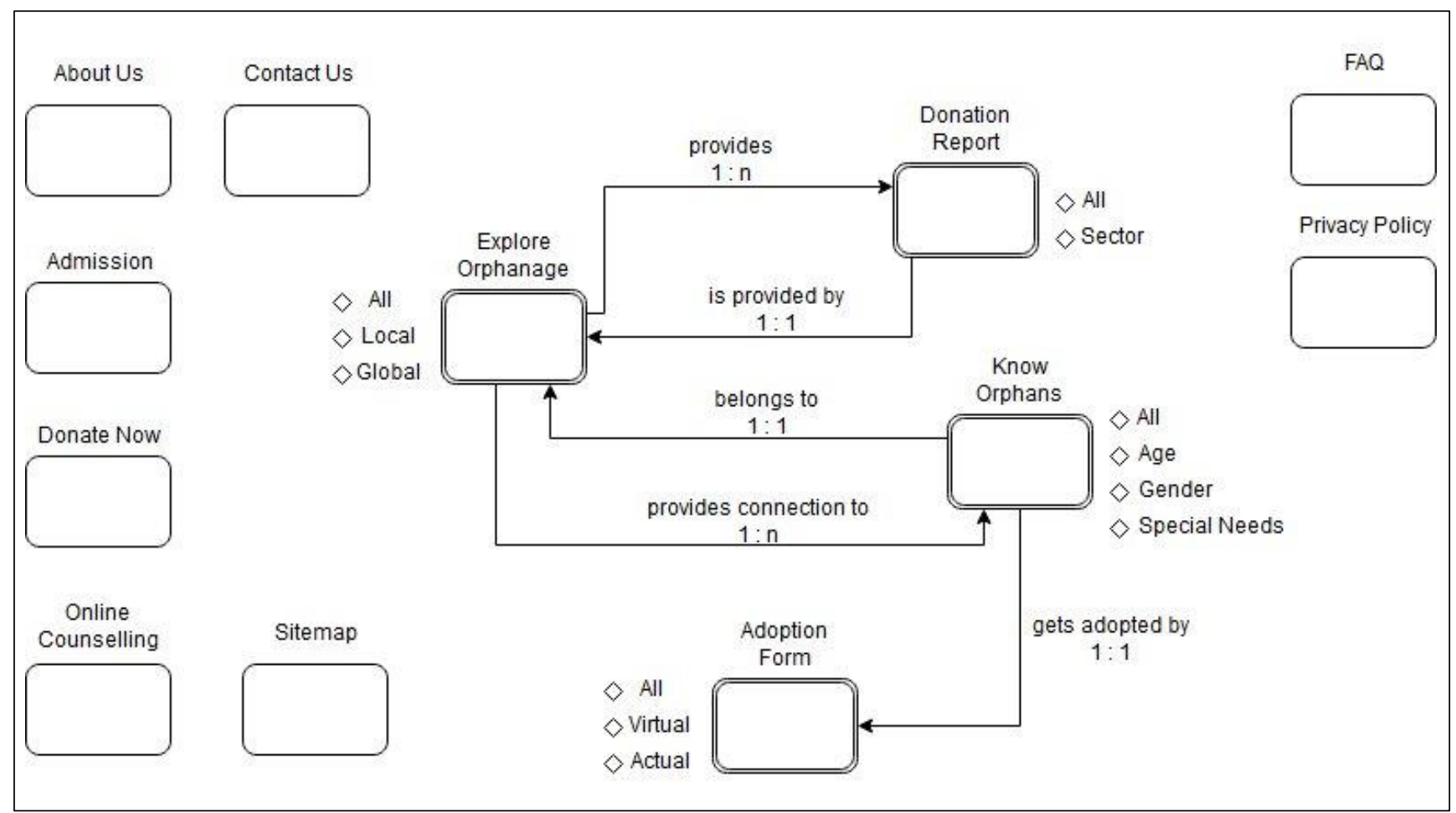

Figure 3: C-IDM of orphanage portal

"provides a connection to". For instance, someone might want to know about donations types of a particular orphanage, and so there should be a relation between Explore Orphanage and Donation Report. Finally, a group of topics is a particular list of topics and a parametric group of topics is a collection of groups of topics. In this design, orphanages could be searched under the topic Explore Orphanage by "all category", by "local category" or by "global category". So, All, Local and Global were indicated as a group of topics for the topic Explore Orphanage. Other groups of topics and the entire schema of C-IDM are represented in Figure 3 with necessary symbols.

\section{ii. Logical IDM (L-IDM)}

Logical IDM consists of three mandatory parameters and an optional design parameter, which include dialogue act, transition act, introductory act and multiple introductory acts (optional) (Bolchini \& Paolini, 2006). The content of a topic is divided into a number of units which is known as a dialogue act. In the orphanage portal website, the dialogue acts for the topic Explore Orphanage were Name, Location, Contact, Gallery, Description, Events and Promotional Activity. The dialogue acts Name and Gallery were underlined which presents the structural strategy of this topic, that is, initially these two dialogue acts were visible on the interface and others can be navigated using a different pattern of structural strategies (see Figure 4). Next, the relevant relation in $\mathrm{C}$-IDM that had cardinality of 1: $\mathrm{n}$ was specified as transition act in L-IDM and the relevant relations having other cardinalities were kept unchanged. A transition act mainly indicates a list of instances when the topic is multiple and the navigation of this list can also be structured using a different pattern of transitional strategy. In this website, there existed a transition act between Explore Orphanage and Know Orphans because a particular orphanage can accommodate a lot of orphans. So, before knowing about an orphan of a particular orphanage, this transition act helps to go through a list of orphans of that orphanage. Finally, a group of topics of C-IDM was specified as an introductory act in L-IDM. Each group of topics is linked to a list of possible instances when the topic is multiple and the navigation of the list can also be structured using a different pattern of subject strategy. In this website, the introductory acts of the topic Know Orphans were indicated as All, Age, Gender and Special Needs, that is before knowing a particular orphans' details, the orphans can be searched "by a list of all category", by "a list of age", by "a list of gender" or by "a list of special needs". The entire schema of L-IDM is represented in Figure 4.

\section{iii. Page IDM (P-IDM)}

Page IDM is the final phase of IDM and involves designing the prototypes for each design parameter discussed above. Figure 5 shows the P-IDM for the homepage of the website orphanage portal. This phase consists of some pages (Bolchini \& Paolini, 2006) where each dialogue act, transition act and the introductory act becomes a page, relevant topics and relevant group of topics become landmarks. For each dialogue act, there should be the content of a kind of topic, structural links of other dialogue acts of the same topic, transition links for transition acts, group of topic links to go from one instance to another, orientation info to indicate the current position of user and landmarks. On the other hand, for a single topic, there is no need for transition links and a group of topic links.

Figure 6 shows a P-IDM for a single topic About Us where the topics' content Aim is displayed by default and contained structural links for Founders' Profile, Our Team and History; the breadcrumbs indicated the orientation info that the users' current position is in Aim inside About Us; the header and footer menu bar and other info links, logo and name are the landmarks. The Explore Orphanage, Know Orphanage, Admission, Adoption Form, Donation Report, and Online Counseling are landmarks used as the header menu, while the About Us, Contact Us, and Donate Now are the landmarks used as a footer menu. 


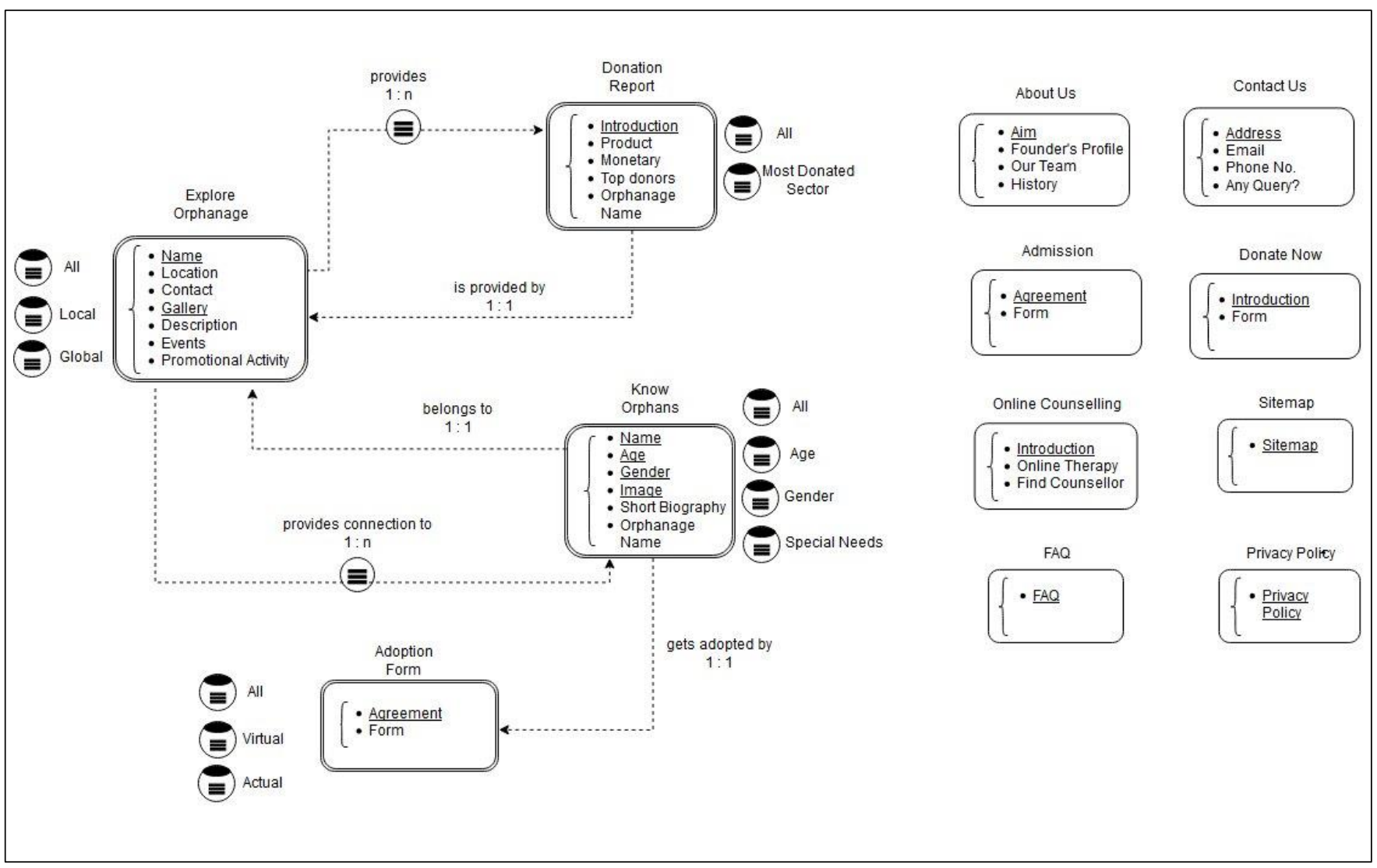

Figure 4: L-IDM of orphanage portal

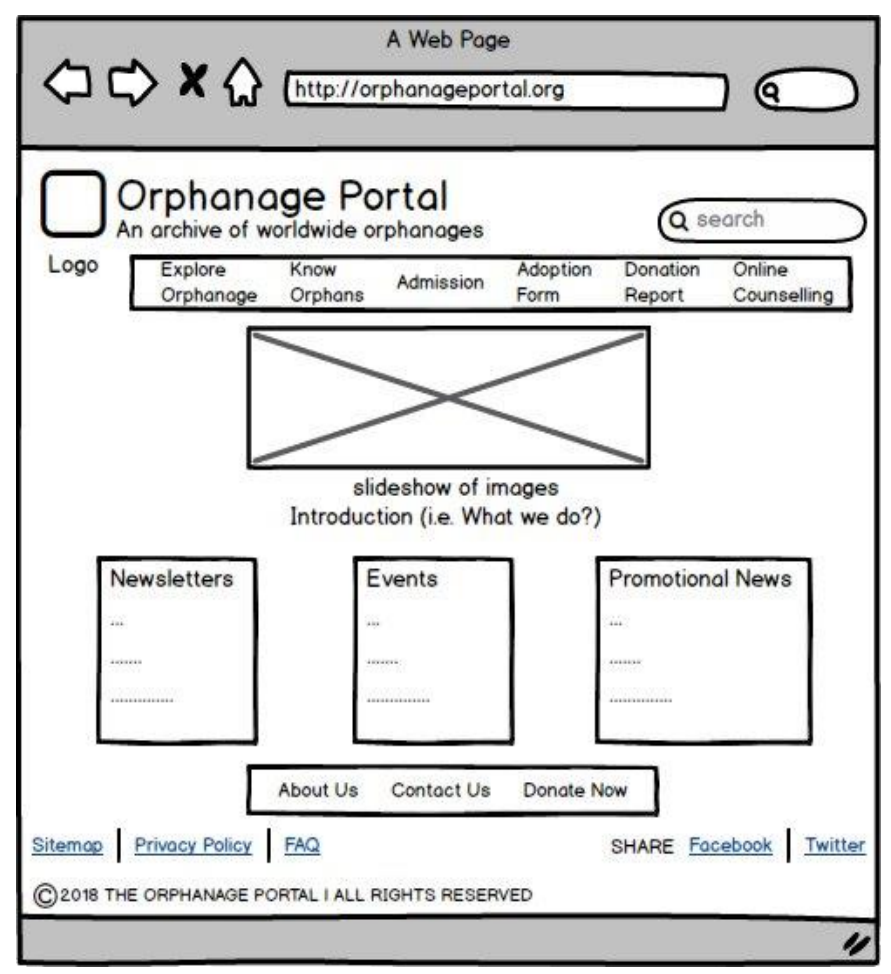

Figure 5: P-IDM of homepage in orphanage portal

Figure 7 shows a P-IDM for a kind of topic Explore Orphanage that is a page displaying the lists for all orphanages by default and links to local and global orphanages list. Selecting a particular orphanage led to a page displaying the topics' content Gallery and Name by default and structural links to other dialogue acts; transition links and the introductory acts were presented as before.

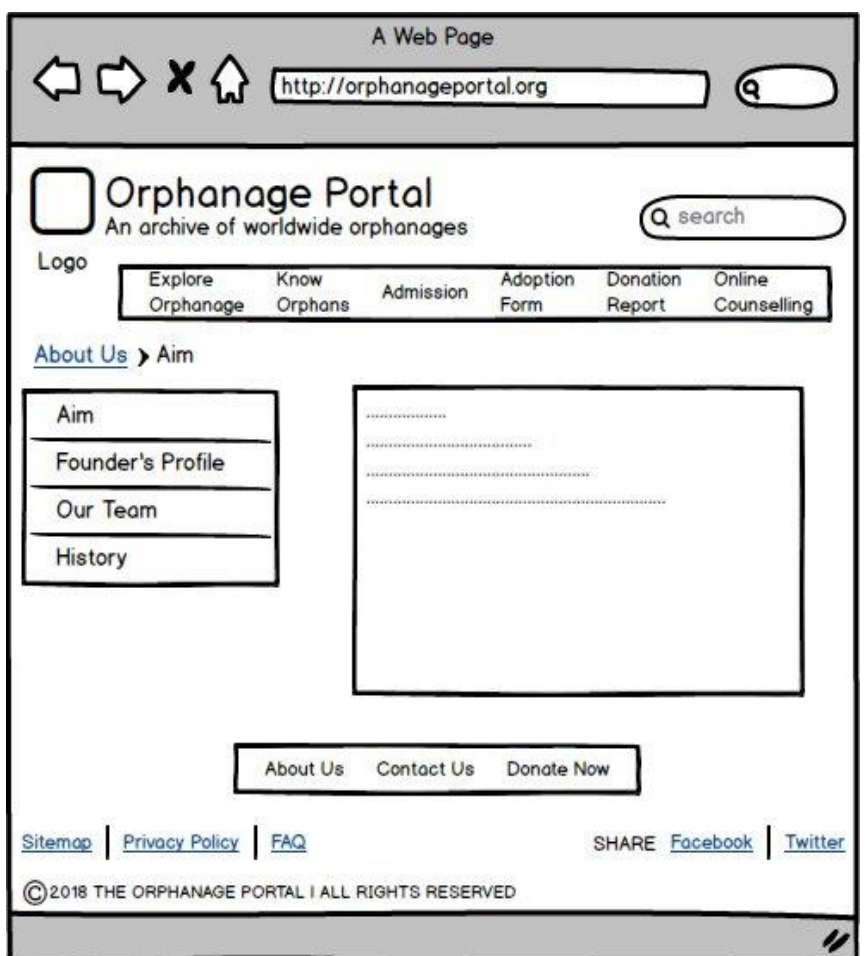

Figure 6: P-IDM of About Us in orphanage portal

There existed a group of topic links on this page to navigate to the next or previous orphanage details. The transition link "connect with orphans" on this page led to a page containing a list of orphans from where a particular orphan can be selected for details and furthermore. The landmarks and orientation info were as before. 

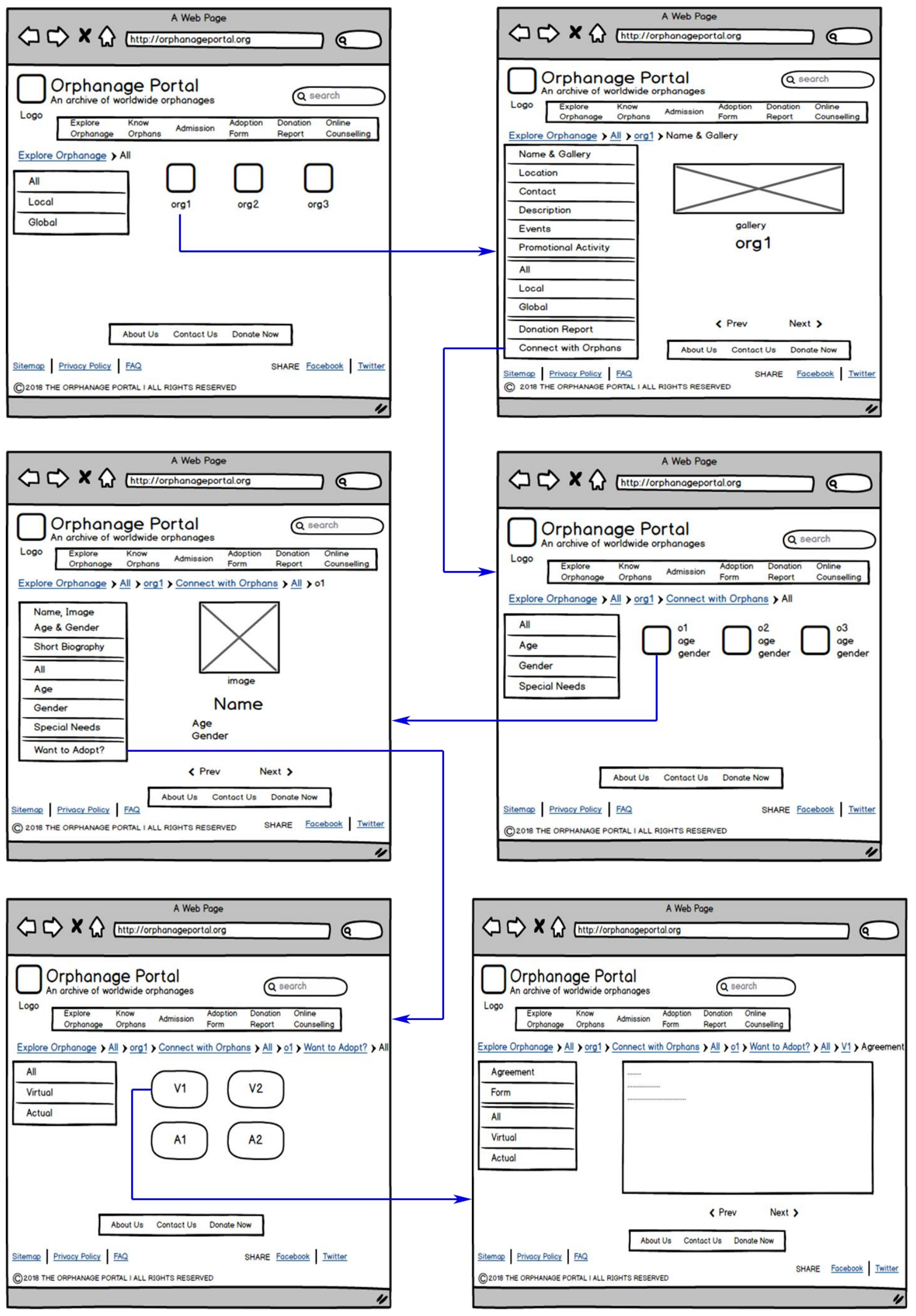

Figure 7: P-IDM steps of Explore Orphanage in orphanage portal 


\section{Development of Web Portals}

For each design, a separate web portal was developed. For the front-end development; HTML, CSS, JavaScript and PHP were used. The Apache server and MySQL database of the XAMPP platform were used to deploy the portals to a local server. Figure 8 shows a user interface of the homepage which has been developed using the Card Sorting design technique. On the other hand, Figure 9 shows a user interface of the homepage which has been developed using the IDM design technique.

\section{COMPARATIVE EVALUATION STUDY}

To evaluate the usability of the two developed websites, an experiment has been conducted. The independent variable of this experiment is the web systems: the website designed using the IDM and the website designed using the card sorting. Dependent variables are some metrics which are chosen to ensure that the goal is reached such as task completion time, success/fail, number of clicks, number of attempts and number of asking help to perform the given task. The experiment has been designed following the within-subjects' concept that means all participants performed the given tasks for both websites. The following subsections describe briefly about the profile of participants, how the experiment has been conducted and the findings of this experiment.

\section{A. Participant Profile}

About fourteen individuals were invited through email and social media to participate in the experiment and ten of them participated in the experiment. There were four female participants and six male participants. The age of the participants varied from 22 to 31 years. All the participants were graduated from various disciplines. All of them had experiences of using the internet and three of them were undergraduate students major in the computer science discipline and others were major in a non-computer science discipline. None of the participants had familiarity with the orphanage system. The participants were assured that their participation is of their own accord and none of their provided information would be used other than experiment purposes. Moreover, participation in the study was completely voluntary and as such, no incentive or compensation has given to the participants.

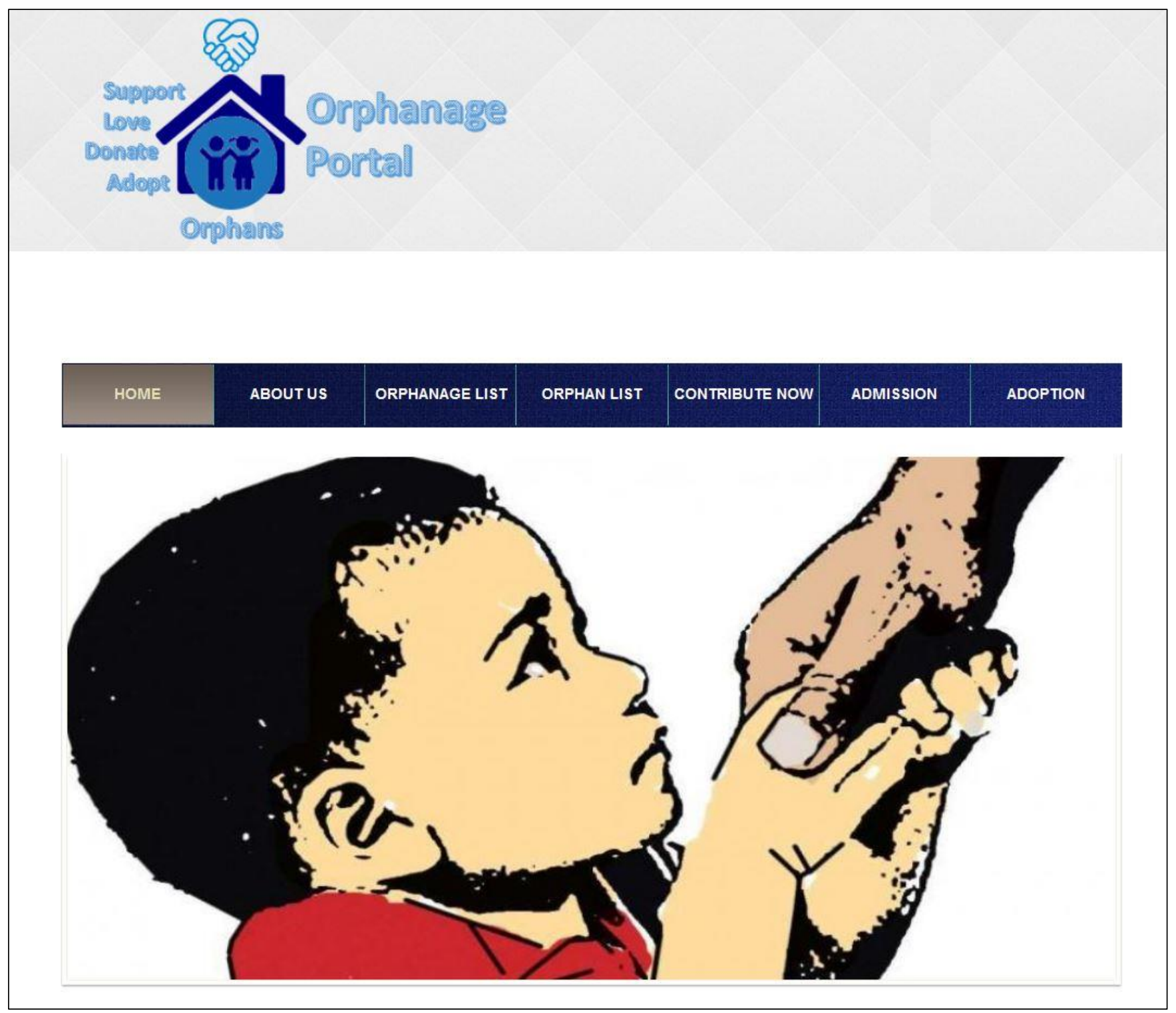

Figure 8: User interface of Homepage of orphanage portal using Card Sorting 


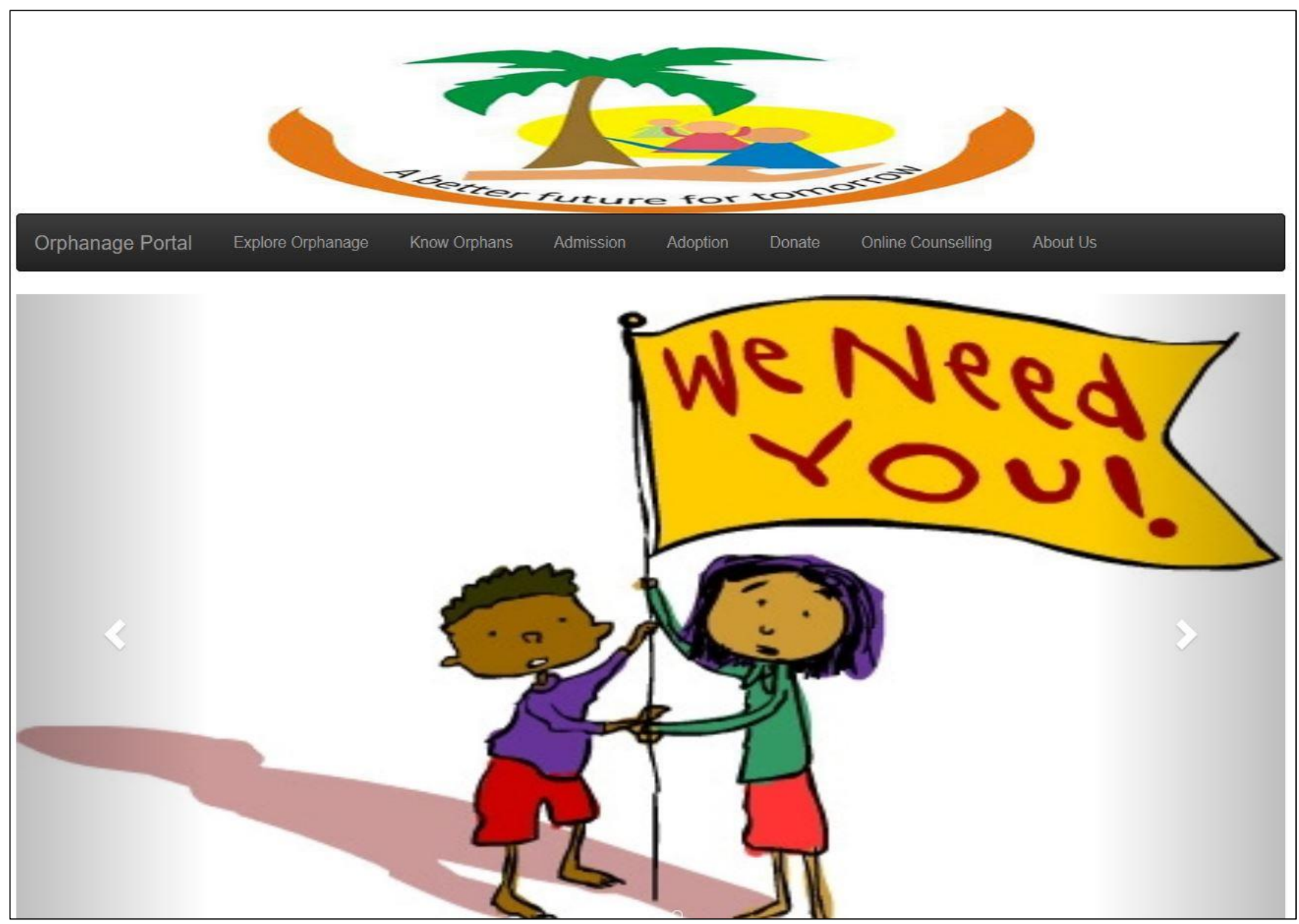

Figure 9: User interface of the Homepage of orphanage portal using IDM

\section{B. Study Procedure}

The experiment was performed in the Software Engineering Laboratory of the authors' institute. A written informed consent was taken from each participant to inform them that participation is voluntary and at any moment they can choose to stop participation in the evaluation study; while the data collected from them will be used anonymously solely for the research and research publications. A total of ten test-sessions were conducted, each with an individual participant. For each test-session, initially, an introduction along with a short demonstration was given to the participants regarding their role and objective of this experiment. After the demonstration, each participant was provided with two sheets - participant's biographical information sheet, and a task sheet followed by a post-test. The tasks were firstly to find details of a local orphanage (Task 01), then to find a female orphan of any local orphanage (Task 02) and finally to adopt the female orphan of that local orphanage that has been found (Task 03). At first, the participants filled up their biographical information sheet and signed the consent form. Then they performed the assigned tasks for each website. While the participants were performing the tasks, required data (data related to the dependent variables) were collected for each participant through observation. After completing the tasks, the participants filled up the post-questionnaire sheet to provide their overall opinion (qualitative feedback) about the experimented websites along with a subjective rating on their overall satisfaction in a range of 1 (strongly dissatisfied) to 5 (strongly satisfied).

\section{Analysis and Findings}

This subsection briefly discusses the analysis and study findings in a different perspective as presented below:

- Number of clicks: The average number of clicks for each task has been calculated for both IDM and card sorting design techniques. The minimum number of clicks required to perform a specific task is considered here as the optimal (number of clicks) value. The difference between the optimal number of clicks and the average number of clicks has been calculated and the deviations are presented in Table 1. It shows that for Task 01 and Task 03, IDM differs slightly less than card sorting but for Task 02 , IDM differs a bit greater than card sorting.

Table 1

Comparison between optimal number of clicks and average number of clicks

\begin{tabular}{cccc|ccc}
\hline \hline & \multicolumn{3}{c}{ IDM } & \multicolumn{3}{c}{ Card Sorting } \\
\cline { 2 - 7 } Tasks & $\begin{array}{c}\text { Optimal } \\
\text { Clicks }\end{array}$ & $\begin{array}{c}\text { Average } \\
\text { Clicks }\end{array}$ & Deviation & $\begin{array}{c}\text { Optimal } \\
\text { Clicks }\end{array}$ & $\begin{array}{c}\text { Average } \\
\text { Clicks }\end{array}$ & Deviation \\
\hline $\begin{array}{c}\text { Task } \\
\mathbf{0 1}\end{array}$ & 3.0 & 3.4 & 0.4 & 2.0 & 2.8 & 0.8 \\
$\begin{array}{c}\text { Task } \\
\mathbf{0 2}\end{array}$ & 2.0 & 2.7 & 0.7 & 2.0 & 2.2 & 0.2 \\
$\begin{array}{c}\text { Task } \\
\mathbf{0 3}\end{array}$ & 2.0 & 2.4 & 0.4 & 3.0 & 3.5 & 0.5 \\
\hline \hline
\end{tabular}


- Number of attempts and asking for help: While the optimal value for the number of attempts is one, the IDM based website showed almost optimal value except Task 02. The Card Sorting based website showed on average 2 to 3 more attempts than the optimal value as shown in Figure 10(a). Again, for each task the number of attempts showed higher value for Card Sorting based website than the IDM based website. Almost similar results are observed in case of number of times asking for help (see Figure 10(b)). Since, the optimal value for the number of asking help is zero, the results showed that none of the tasks was completed with optional (number of times asking for help) value for both websites. Again, the average number of times asking for help does not vary for Task 01 for both design techniques, while the Card Sorting based website differs most in the case of Task 03 and slightly greater for Task 02 comparing to the IDM based website.

- Task completion time: The participants were able to complete each task successfully for both websites. The TCT for the assigned tasks varied from 8 seconds to 24 seconds for IDM based website, while for the Card Sorting based website the TCT was 12 seconds to 26 seconds. That is, on average, the IDM based website taken comparatively less task completion time (TCT) to complete each of the assigned tasks than the Card Sorting based website (see Figure 10(c)).

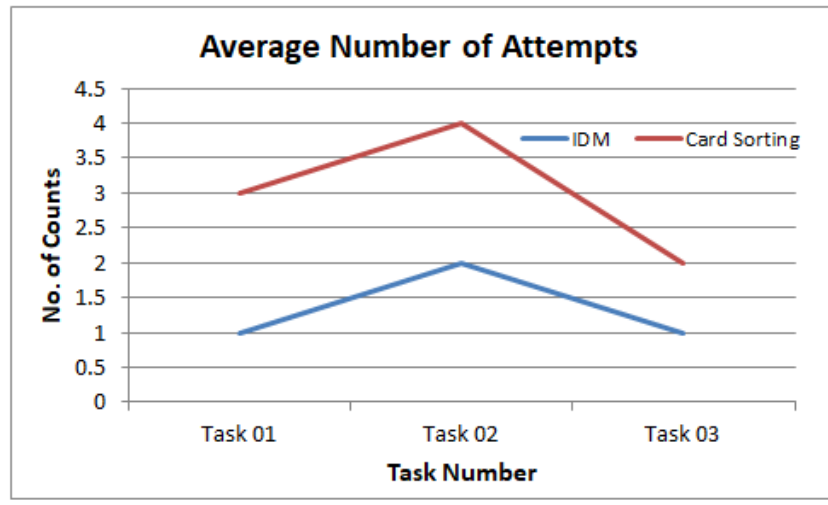

(a) Average number of attempts

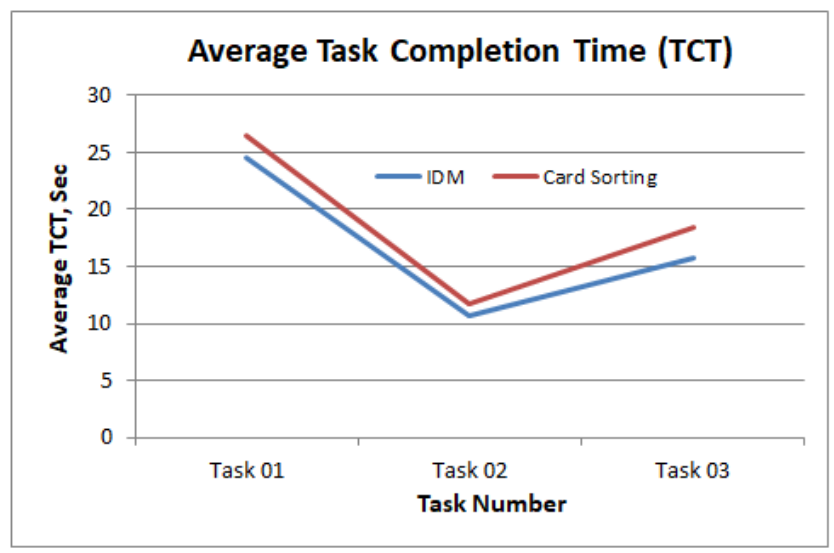

(c) Average task completion time
- Overall satisfaction scores and qualitative feedback: The overall satisfaction score rated by the participants has got much support for the website designed by using IDM than the website designed using card sorting (see Figure 10(d)). As qualitative feedback, participants recommend some features that can enhance the usability of the websites in the future. For the web site designed using IDM, the participants suggested that the icons should be more user-friendly and the interface should be attractive, Google Map should be integrated to find the location of an orphanage. They have commented that the website provides the required functionality to perform the given tasks but it is a little complex to use. On the other hand, for the website designed using card sorting, the participants suggested that the navigation system for the list of the orphanage and orphan information requires much improvement, different pages of the website should be linked up in a user-friendly way, Google Map should be integrated to find the location of an orphanage and necessary information of a child should be provided on the adoption page. They have also agreed to the fact that such a website for an orphanage is helpful and trustworthy for the adoption process. Moreover, most of the participants recommend the IDM design technique over card sorting for designing such an informative website in the future.

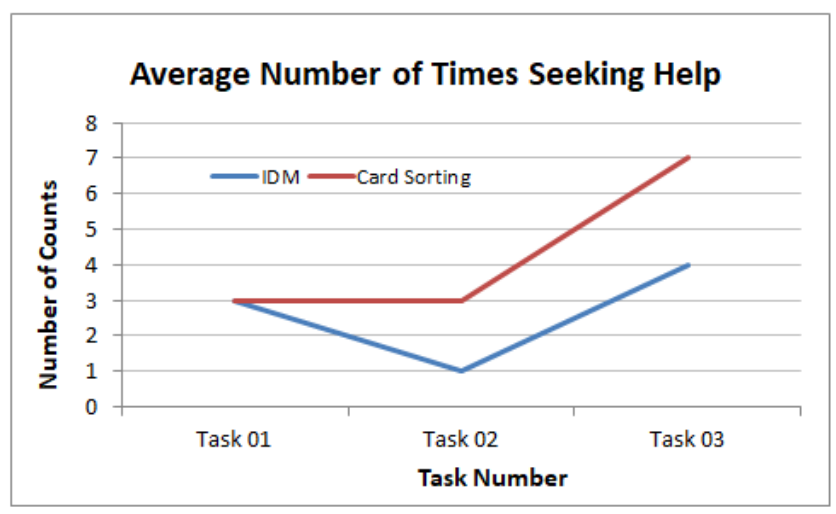

(b) Average number of times asking help

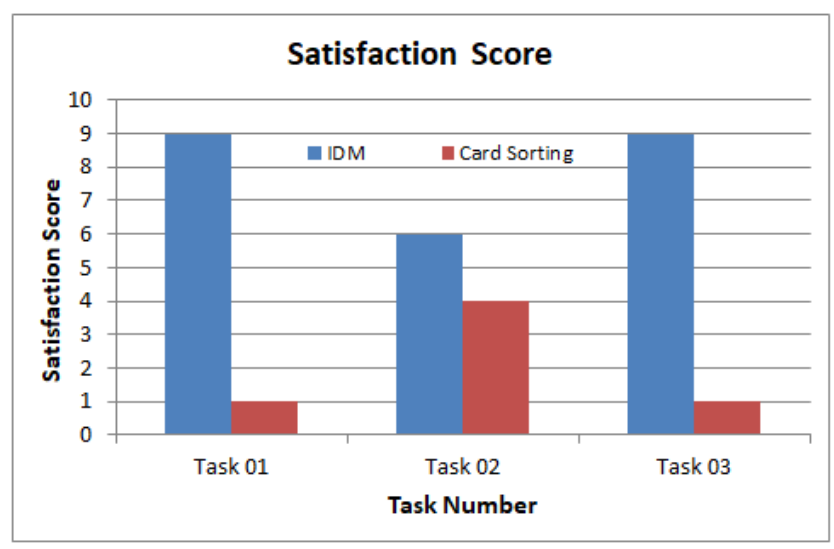

(d) Satisfaction score

Figure 10: Graphical representation of chosen metrics for Task 01, Task 02 and Task 03 


\section{CONCLUSIONS}

This research showed that the IDM based websites performed well than the Card Sorting based website from the usability perspective. The outcome of this research thus contributes to the HCI practitioners by making them aware that the UX and usability of a website depend on an appropriate selection of a design technique to design and develop the website; and by showing them that the IDM is performed better in design information-intensive website. Again, while the existing research mainly focused on the UX and usability evaluation (Park et al., 2020), and comparing the performance of different UX and evaluation methods (Ternauciuc \& Vasiu, 2015), this research explored a new concept, which was not only the UX and usability evaluation approach but also the design choice had a strong impact on the development of a usable website for end-users. However, this research had a few limitations. The number of participants was not adequate from whom the data were collected. Since an orphanage portal website was developed, and the assigned task includes the adoption process of orphans, so the participants should have been adults who are interested in child adoption or are concerned of children. But, most of the participants were not as desired. Again, only one website (i.e., the orphanage web system) was developed here as an experiment purpose, developing multiple information-intensive websites would give more generalize outcomes. Moreover, to analyze the evaluation data, the concept of Fitts' law was not considered to assess the number of clicks with respect to the time to measure the RMS values. The future work of this study would be firstly, to develop the concrete versions of the websites by including more features or functionality, and by making the websites dynamic in nature. Secondly, to conduct a further experiment with more desired participants and conducting an in-depth analysis of the study data to measure more usability factors. Thirdly, potential future research may consider other available design techniques to compare with the CS and IDM techniques to provide a more generalize and effective outcome for the HCI practitioners.

\section{ACKNOWLEDGEMENTS}

The authors would like to acknowledge the contribution of the participants who have voluntarily participated in the experiment of this research work.

\section{REFERENCES}

Abran, A., Khelifi, A., Suryn, W., \& Seffah, A. (2003). Usability meanings and interpretations in ISO standards. Software Quality Journal, 11(4), 325-338.

Al-Fadhli, S. (1997). Critical success factors influencing ECommerce in Kuwait. Journal of Internet Banking and Commerce, 16(1), 1-7.

Bolchini, D. \& Garzotto, F. (2008). Designing multichannel web applications as "Dialogue Systems": The IDM Model. In: Rossi G., Pastor O., Schwabe D., Olsina L. (Eds.), Web Engineering: Modelling and Implementing Web Applications. (pp. 193-219). London, United Kingdom: Springer-Verlag.

Bolchini, D., \& Garzotto, F. (2007, December 3-7). Quality of web usability evaluation methods: An empirical study on MiLE+. Proceedings of the International Conference on Web Information Systems Engineering (WISE'07), Berlin, Heidelberg. (pp. 481-492).

Bolchini, D., \& Paolini, P. (2006). Interactive dialogue model: A design technique for multichannel applications. Journal of IEEE Transactions on Multimedia, 8(3), 529-541.
Brejcha, J., \& Marcus, A. (2013, July 21-26). Semiotics of interaction: Towards a UI alphabet. Proceedings of the 15th International Conference on Human-Computer Interaction (HCII'13), Las Vegas, Nevada, USA. (pp. 13-21).

Conte, T., Massollar, J., Mendes, E., \& Travassos, G. H. (2007, September 20-21). Usability evaluation based on web design perspectives. Proceedings of the 1st international symposium on Empirical Software Engineering and Measurement (ESEM '07), Madrid, Spain. (pp. 146-155).

Coppola, G., Fiore, A., Mainetti, L. \& Pandurino, A. (2015, June 23-26). Mobile-IDM: A design method for modeling the new interaction style of mobile applications. Proceedings of the 15th International Conference on Web Engineering (ICWE'15), Rotterdam, Netherlands. (pp. 101-115).

Craske, D. J. (2010). Conceptual, logical \& page designs for a proposed website for golf courses of Central Indiana and Indiana Microbrews [Technical report], Indiana University School of Informatics, Indiana, USA.

Duncan, J., \& Holliday, W. (2008). The role of information architecture in designing a third-generation library website. Journal of College and Research Libraries, 69(4), 301-318.

Ebenezer, C. (2003). Usability evaluation of an NHS library website. Health Information and Libraries Journal, 20(3), 134-142.

Faiks, A., \& Hyland, N. (2000). Gaining user insight: A case study illustrating the card sort technique. Journal of College and Research Libraries, 61(4), 350-357.

French, T., Liu, K., \& Springett, M. (2007, September 3-7). A cardsorting probe of E-Banking trust perceptions. Proceedings of the 21st British HCI Group Annual Conference on People and Computers (BCS-HCI'07), University of Lancaster, United Kingdom. (pp. 45-83).

Garzotto, F., Paolini, P., \& Schwabe, D. (1993). HDM-a modelbased approach to hypertext application design. Journal of ACM Transactions on Information Systems (TOIS), 11(1), 126.

Gray, W. D., \& Salzman, M. C. (1998). Damaged merchandise? a review of experiments that compare usability evaluation methods. Journal of Human-Computer Interaction, 13(3), 203-261.

Islam, M. N., \& Bouwman, H. (2016). Towards user-intuitive web interface sign design and evaluation: A semiotic framework. International Journal of Human-Computer Studies, 86, 121137.

Islam, M. N., Oishwee, S. J., Mayem, S. Z., Mokarrom, A. S. M. N., Razzak, M. A., \& Kabir, A. B. M. H. (2017, December 79). Developing a multi-channel military application using Interactive Dialogue Model (IDM). Proceedings of the 3rd International Conference on Electrical Information and Communication Technology (EICT'17), Khulna, Bangladesh. (pp. 1-6).

Islam, M. N., Bouwman, H. \& Islam, A. K. M. N. (2020). Evaluating Web and Mobile User Interfaces with Semiotics: An Empirical Study. IEEE Access, 8(1), 84396-84414. DOI: 10.1109/ACCESS.2020.2991840

Jeffries, R., Miller, J. R., Wharton, C., \& Uyeda, K. (1991, April 27-May 2). User interface evaluation in the real world: A comparison of four techniques. Proceedings of the SIGCHI Conference on Human factors in Computing Systems (CHI'91), New Orleans, Louisiana, USA. (pp. 119-124).

Karat, C. M., Campbell, R., \& Fiegel, T. (1992, June 3-7). Comparison of empirical testing and walkthrough methods in user interface evaluation. Proceedings of the SIGCHI Conference on Human factors in Computing Systems (CHI'92), Monterey, California, USA. (pp. 397-404).

Kumar, B. A., \& Goundar, M. S. (2019). Usability heuristics for mobile learning applications. Education and Information Technologies, 24(2), 1819-1833.

Kurniawan, S., \& Zaphiris, P. (2005, October 9-12). Researchderived web design guidelines for older people. Proceedings of the 7th International ACM SIGACCESS Conference on 
Computers and Accessibility (Assets'05), Baltimore, Maryland. (pp. 129-135).

Marcus, A. (Ed.) (2015). Design, User Experience, and Usability: Users and Interactions. Proceedings of the $4^{\text {th }}$ International Conference, DUXU 2015, Held as Part of HCI International 2015, Los Angeles, CA, USA, August 2-7, 2015, Proceedings (Vol. 9187), Springer.

Matera, M., Costabile, M. F., Garzotto, F., \& Paolini, P. (2002). SUE inspection: An effective method for systematic usability evaluation of hypermedia. Journal of IEEE Transactions on Systems, Man, and Cybernetics-Part A: Systems and Humans, 32(1), 93-103.

Mubin, S. A., Jantan, A. H. Jantan, \& R. Abdullah (2015, December 16-17). UML-based navigational design approach for modeling complex interactions in web applications. Proceedings of the $9^{\text {th }}$ Malaysian Software Engineering Conference (MySEC'15), Kuala Lumpur, Malaysia. (pp. 6063).

Nielsen, J. (1995). 10 usability heuristics for user interface design. (Extracted on Apr. 23, 2020). Source: https://www.nngroup.com/articles/ten-usability-heuristics.

Park, K., Jeong, M., \& Kim, K. (2020). Usability evaluation of menu interfaces for smartwatches. Journal of Computer Information Systems, 60(2), 156-165.

Palmer, J. W. (2002). Web site usability, design, and performance metrics. Journal of Information Systems Research, 13(2), 151167.

Perrone, V., Bolchini, D., \& Paolini, P. (2005, September 21-23). A stakeholders centered approach for conceptual modeling of communication-intensive applications. Proceedings of the 23rd Annual International Conference on Design of Communication: Documenting \& Designing for Pervasive Information (SIGDOC'05), Coventry, United Kingdom. (pp. 25-33).

Petrie, H., Power, C., Cairns, P., \& Seneler, C. (2011, September 59). Using card sorts for understanding website information architectures: Technological, methodological and cultural issues. Proceedings of the $13^{\text {th }}$ IFIPTC13 International Conference on Human-Computer Interaction (INTERACT'11), Lisbon, Portugal. (pp. 309-322).

Preece, J., Rogers, Y., \& Sharp, H. (2004). What is interaction design? In S. Kurniawan (Ed.), Interaction design: Beyond human-computer interaction (Vol. 3, pp. 1-18). Sussex, United Kingdom: John Wiley \& Sons Ltd.
Razzak, M. A., \& Islam, M. N. (2020). Exploring and Evaluating the Usability Factors for Military Application: A Road Map for HCI in Military Applications. Human Factors and Mechanical Engineering for Defense and Safety, 4(4), 1-18.

Rabi'u, S., Ayobami, A. S., \& Hector, O. (2012, November 2). Usability characteristics of mobile applications. Proceedings of First International Conference on Behavioural \& Social Science Research (ICBSSR), Kampar, Malaysia. (pp. 1-5).

Rugg, G. \& McGeorge, P. (2005). The sorting techniques: A tutorial paper on card sorts, picture sorts and item sorts. Journal of Expert Systems, 22(3), 94-107.

Sagar, K., \& Saha, A. (2017). A systematic review of software usability studies. International Journal of Information Technology, 9 (4), 1-24. DOI: 10.1007/s41870-017-0048-1

Spagnolo, L., Bolchini, D., Paolini, P., \& Blas, N.D. (2010). Search-enhanced information architecture for contentintensive rich internet applications. Journal of Information Architecture, 2(1), 19-36.

Spencer, D. (2009). Card sorting: Designing usable categories. Rosenfeld Media, Brooklyn, New York.

Ternauciuc, A., \&Vasiu, R. (2015). Integrated usability testing. TEM Journal, 4(4), 388 -395.

Tullis, T. S. (2007). Using closed card-sorting to evaluate information architectures. Proceedings of the Usability Professionals Association (2007) Conference, Austin, TX. (pp. 11-15).

Troyer, O. D., \& Leune, C. J. (1998). WSDM: A user-centered design method for web sites. Journal of Computer Networks and ISDN Systems, 30 (1-7), 85-94.

Wentzel, J., Müller, F., Beerlage-de Jong, N., \& van GemertPijnen, J. E. W. C. (2016). Card sorting to evaluate the robustness of the information architecture of a protocol website. International Journal of Medical Informatics, 86, 7181.

Zhang, T., Rau, P. L. P., \& Salvendy, G. (2010). Exploring critical usability factors for handsets. Behaviour \& Information Technology, 29(1), 45-55.

Zimmerman, D. E., \& Akerelrea, C. (2002, September 17-20). A group card sorting methodology for developing informational websites. Proceedings of the International Professional Communication Conference (IPCC'02), Portland, Oregon, USA. (pp. 437-445). 